\title{
Puzzling rotation of asteroid 288 Glauke
}

\author{
A. Kryszczyńska, T. Kwiatkowski, and T. Michałowski
}

Astronomical Observatory, Adam Mickiewicz University, Słoneczna 36, 60-286 Poznań, Poland

Received 9 January 2003 / Accepted 13 March 2003

\begin{abstract}
Most asteroids rotate about their axis of maximum moment of inertia. However, for several small asteroids with very slow rotation rates, the damping time scale is expected to be longer than the age of the Solar System, implying that these objects may exhibit non-principal axis rotation. The asteroid 288 Glauke is suspected to undergo tumbling rotation because of very long period of light variations. We observed this object during 39 nights from January to May 2000 at the Borowiec Observatory (Poland). Our lightcurve with a peak-to-peak amplitude of 0.7 mag has a complicated nature which can result from a non-principal axis rotation or a very complex shape of this body.
\end{abstract}

Key words. techniques: photometric - minor planets, asteroids

\section{Introduction}

Photometric observations of asteroids show that most of them are in a state of principal-axis rotation. According to theories (Harris 1994) more complex modes of rotation induced by mutual collinsions can be expected only for small objects with relatively long periods of rotation.

The largest known slow rotator is a C-type asteroid 253 Mathilde, which has a rotation period of 17.41 days and a mean diameter of $53 \mathrm{~km}$. Observations of this asteroid revealed a secondary period which can be interpreted as evidence of its complex rotation state (Mottola et al. 1995; Veverka et al. 1997).

The best-studied slow rotator is a near-Earth S-type asteroid 4179 Toutatis. Both radar (Hudson \& Ostro 1995) and photometric (Spencer et al. 1995) observations of this object have confirmed that it is in a long axis precession mode with periods of 7.42 days (long-axis precession about the angular momentum vector) and 5.37 days (rotation about long axis), (Ostro et al. 1999).

Lightcurves of several other asteroids with long periods show deviations from single periodicity that may suggest that these bodies are in a nonprincipal axis (NPA) rotation state. There include 1689 Floris-Jan (Harris 1994), 3288 Seleucus (Harris et al. 1999), 3691 Bede and 1997 BR (Pravec et al. 1998) as well as 1999 GU3 (Pravec et al. 2000). The last one has a diameter of about $0.5 \mathrm{~km}$ and is the smallest of all known slow rotators.

288 Glauke is another example of a slow rotating object. It is an S-type asteroid with an effective diameter of $32 \mathrm{~km}$ and absolute magnitude $H=9.84 \mathrm{mag}$ (http://pdssbn.astro.umd.edu). As a member of the

Send offprint requests to: A. Kryszczyńska, e-mail: agn@amu.edu.pl
Main Belt, Glauke can be observed approximately every 1.5 year. Due to its long period of light variation, the best times for its observation fall during its stationary points, when the asteroid can be observed for many nights in the same star field (Nakamura \& Fuse 1998).

\section{Previous observations}

The first photometric observations of Glauke were performed by Harris et al. (1999) who observed it from April to June 1982. Since the asteroid never changed its brightness by more then $0.05 \mathrm{mag}$ in the course of a night, they combined single measurements to form a weighted average, and their final lightcurve consisted of 20 normal points. Those observations allowed them to determine a period of light variation, whose value depended on the adopted $G$ for the solar phase angle correction. As a result, the final period lay within a range from 1110 to 1210 hours (46 to 50 days) - the longest ever observed for any asteroid.

The paper by Harris et al. (1999) includes also results of the photographic photometry of Glauke performed in August/September 1983 at Lowell Observatory. It consists of six points only and does not add anything new to the previous data apart from a confirmation of the long period.

Binzel (1987) observed Glauke from September to November 1984 and even though his lightcurve had only 9 points (two of them were average values from several measurements), obtained a period of approximately 50 days. He did not find any indication of shorter period variations. Harris et al. (1999) reanalysed Binzel's data and derived a period of rotation of 1296 hours (54 days). This period was inconsistent with the period determined from 1982 observations no matter what $\mathrm{G}$ value was chosen. The most likely reason for that discrepancy was that 288 Glauke is in a NPA rotation spin state. 
New data obtained on five consecutive nights in January 1986 by Weidenschilling et al. (1990) were used to determine the photometric behaviour of Glauke at small phase angles. They detected a significant opposition effect for this body.

In March 2000 Glauke was observed with the Arecibo radar by Ostro et al. (2001). The echo spectra they obtained on six days in March 2000 were rather noisy, so only the global physical parameters of this asteroid could be obtained. It appeared that Glauke is rather smooth at cm-to-m scales and its radar properties are similar to the mean values of the radar properties of other main-belt S-type asteroids. The period of rotation was found to be shorter than 2100 hours but it was not possible to give a more accurate value for it.

As the previously obtained lightcurves are rather sparse, it is not possible to definitely check whether Glauke has a relaxed or complex rotation. In order to answer this question we decided to collect photometric data on this asteroid spread over two previously determined periods.

\section{CCD photometry in the 2000 apparition}

During its spring opposition in 2000 Glauke provided a good opportunity for observers from the northern hemisphere. We observed it extensively on 39 nights, from 6th January to 7th May 2000, using a $0.4 \mathrm{~m}$ reflector with the KAF 400 CCD camera located at Borowiec Observing Station of A. Mickiewicz Univeristy, Poznan (Poland). The aspect data for all observing nights are collected in the Table 1 .

In order to achive a good signal to noise ratio, most CCD frames were exposed through a "clear filter" $(C)$, with occasional $R$ and $V$ filter frames being used as a check of the star colours. Normally such unfiltered frames introduce additional biases resulting from the colour-dependent extinction. In our case, however, the spectral response of the KAF-400 chip made our unfiltered measurements close to some rather broad, non-standard $R$ filter magnitudes (Henden 2000), which are less sensitive to different star colours.

A standard reduction of the CCD frames as well as the aperture photometry were performed with the CCLRS STARLINK package. From 6 to 13 January and from 3 to 10 April Glauke was near stationary points on the sky, so we were able to use the same comparison stars.

To connect different star fields in which Glauke was observed we used methods of equal altitudes and overlapping fields. In the former, comparison stars from different nights were observed at equal airmasses which was not difficult since most of them were separated by no more than a few degrees. Such measurements were repeated several times and the scatter of results showed the accuracy of the order of $0.02-0.03 \mathrm{mag}$. In the latter method, overlapping fields allowed us to connect those comparison stars that were not more then one degree apart. Although this approach was based on accurate relative photometry measurements, cummulation of errors made the final accuracy drop to about $0.03-0.05$ mag.

Since all our measurements were performed with the same photometric system, transformation of the obtained magnitudes to the standard Kron-Cousins system was not crucial. On the other hand, such transformation would not be trivial due to the
Table 1. Aspect data.

\begin{tabular}{|c|c|c|c|c|c|c|}
\hline \multirow[t]{2}{*}{ Date (UT) } & \multirow{2}{*}{$\begin{array}{c}r \\
(\mathrm{AU})\end{array}$} & \multirow{2}{*}{$\begin{array}{c}\Delta \\
(\mathrm{AU})\end{array}$} & \multirow{2}{*}{$\begin{array}{c}\text { Phase } \\
\text { angle } \\
\left(^{\circ}\right)\end{array}$} & \multicolumn{2}{|c|}{$\begin{array}{l}\lambda \quad \beta \\
(\mathrm{J} 2000)\end{array}$} & \multirow{2}{*}{$\begin{array}{c}C \\
\text { (mag) }\end{array}$} \\
\hline & & & & $\left({ }^{\circ}\right)$ & $\left({ }^{\circ}\right)$ & \\
\hline 2000 Jan. 06.1 & 2.420 & 1.698 & 18.9 & 158.0 & 2.0 & 9.01 \\
\hline 2000 Jan. 07.0 & 2.418 & 1.686 & 18.7 & 158.0 & 2.0 & 9.06 \\
\hline 2000 Jan. 12.1 & 2.408 & 1.628 & 17.4 & 158.0 & 2.2 & 8.81 \\
\hline 2000 Jan. 13.1 & 2.406 & 1.616 & 17.1 & 158.0 & 2.2 & 8.75 \\
\hline 2000 Jan. 14.0 & 2.404 & 1.605 & 16.8 & 158.0 & 2.3 & 8.70 \\
\hline 2000 Jan. 20.0 & 2.393 & 1.543 & 14.9 & 157.7 & 2.5 & 8.47 \\
\hline 2000 Feb. 12.1 & 2.349 & 1.375 & 5.1 & 154.3 & 3.6 & 7.68 \\
\hline 2000 Feb. 12.9 & 2.348 & 1.371 & 4.7 & 154.1 & 3.6 & 7.63 \\
\hline 2000 Mar. 04.0 & 2.313 & 1.340 & 6.2 & 149.6 & 4.3 & 7.83 \\
\hline 2000 Mar. 05.8 & 2.309 & 1.342 & 7.1 & 149.2 & 4.3 & 7.93 \\
\hline 2000 Mar. 15.9 & 2.293 & 1.371 & 12.0 & 147.3 & 4.5 & 7.94 \\
\hline 2000 Mar. 21.9 & 2.284 & 1.398 & 14.7 & 146.5 & 4.6 & 8.13 \\
\hline 2000 Mar. 22.9 & 2.283 & 1.404 & 15.1 & 146.3 & 4.6 & 8.23 \\
\hline 2000 Mar. 23.9 & 2.281 & 1.409 & 15.5 & 146.2 & 4.6 & 8.33 \\
\hline 2000 Mar. 26.8 & 2.277 & 1.425 & 16.6 & 146.0 & 4.6 & 8.32 \\
\hline 2000 Apr. 03.9 & 2.265 & 1.481 & 19.5 & 145.6 & 4.6 & 8.27 \\
\hline 2000 Apr. 05.0 & 2.264 & 1.488 & 19.8 & 145.6 & 4.6 & 8.32 \\
\hline 2000 Apr. 05.8 & 2.263 & 1.494 & 20.0 & 145.6 & 4.6 & 8.37 \\
\hline 2000 Apr. 06.8 & 2.262 & 1.501 & 20.3 & 145.6 & 4.6 & 8.45 \\
\hline 2000 Apr. 07.9 & 2.260 & 1.511 & 20.7 & 145.6 & 4.6 & 8.51 \\
\hline 2000 Apr. 08.9 & 2.258 & 1.519 & 21.0 & 145.6 & 4.6 & 8.60 \\
\hline 2000 Apr. 09.9 & 2.257 & 1.527 & 21.3 & 145.6 & 4.6 & 8.70 \\
\hline 2000 Apr. 10.8 & 2.256 & 1.533 & 21.5 & 145.7 & 4.6 & 8.80 \\
\hline 2000 Apr. 16.9 & 2.248 & 1.584 & 23.0 & 146.1 & 4.6 & 8.90 \\
\hline 2000 Apr. 17.8 & 2.247 & 1.593 & 23.2 & 146.1 & 4.6 & 8.94 \\
\hline 2000 Apr. 18.8 & 2.246 & 1.602 & 23.4 & 146.2 & 4.6 & 9.01 \\
\hline 2000 Apr. 20.8 & 2.243 & 1.620 & 23.9 & 146.5 & 4.6 & 9.09 \\
\hline 2000 Apr. 21.8 & 2.242 & 1.630 & 24.1 & 146.6 & 4.6 & 9.07 \\
\hline 2000 Apr. 24.8 & 2.239 & 1.658 & 24.6 & 147.0 & 4.6 & 8.95 \\
\hline 2000 Apr. 26.8 & 2.236 & 1.677 & 24.9 & 147.3 & 4.6 & 8.82 \\
\hline 2000 Apr. 28.9 & 2.234 & 1.696 & 25.3 & 147.6 & 4.5 & 8.67 \\
\hline 2000 Apr. 29.9 & 2.233 & 1.706 & 25.4 & 147.8 & 4.5 & 8.58 \\
\hline 2000 Apr. 30.9 & 2.232 & 1.715 & 25.5 & 147.9 & 4.5 & 8.62 \\
\hline 2000 May 1.8 & 2.230 & 1.725 & 25.7 & 148.1 & 4.5 & 8.62 \\
\hline 2000 May 2.8 & 2.229 & 1.735 & 25.8 & 148.3 & 4.5 & 8.65 \\
\hline 2000 May 3.9 & 2.228 & 1.745 & 25.9 & 148.5 & 4.5 & 8.70 \\
\hline 2000 May 5.8 & 2.226 & 1.764 & 26.2 & 148.9 & 4.5 & 8.79 \\
\hline 2000 May 6.8 & 2.225 & 1.775 & 26.3 & 149.2 & 4.4 & 8.84 \\
\hline 2000 May 7.9 & 2.223 & 1.788 & 26.4 & 149.4 & 4.4 & 8.92 \\
\hline
\end{tabular}

"clear filter" used for most observations. Attempts to connect the January stationary point star field with that of April failed as both areas were 15 degrees apart and the atmosphere was not stable enough to properly determine the extinction coefficients. 


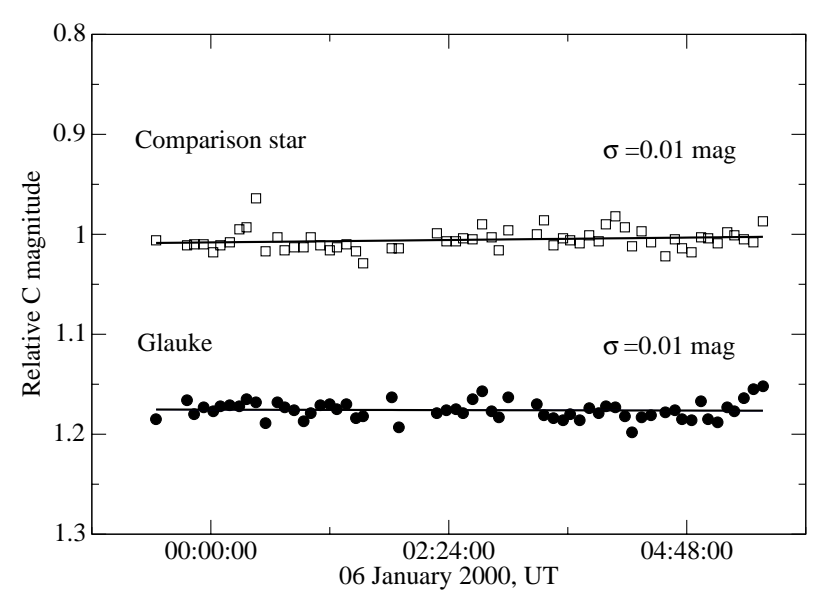

Fig. 1. Single night lightcurve of 288 Glauke. Filled circles represent relative brightness of the asteroid, empty squares relative brightness of a comparison star.

\section{Results}

Single night observations of 288 Glauke did not reveal any short period light variations. An example of such one-night lightcurves obtained during 6 hours on 6th January 2000 can be seen in Fig. 1. Within the statistical error of $0.01 \mathrm{mag}$, the brightness of the asteroid was constant throughout the time of observations.

To study the long-time light variations of Glauke we computed one normal point for each night. Results are presented in Fig. 2 showing "clear filter" magnitudes corrected for varying asteroid-Sun and asteroid-Earth distances as well as the solar phase angle. The latter correction was done with $G=0.23$, the mean value for S-type, Main Belt asteroids. To show the influence of $G$ on the results, two other lightcurves are shown for the upper $(G=0.34)$ and lower $(G=0.12)$ uncertainties on the average $G$. The observed relative "clear filter" magnitudes C without the mentioned above corrections are in the last column of Table 1.

The maximum peak-to-peak amplitude of the lightcurve is about 0.7 mag. It is calculated from the April 20 minimum and May 1 maximum, between which the viewing and illumination geometries did not change by more than 2-3 degrees.

The lightcurve consists of two parts that are not connected in magnitude due to the lack of common comparison stars. However, they can be overlapped using characteristic features existing on both parts. This requires the assumption of principal axis rotation. In such a case the only possible period for the composite lightcurve would be $77 \pm 0.5$ days ( $1848 \pm 12$ hours) - see Fig. 3.

This period is longer than the values determined previously by Harris et al. (1999) from their own data - 1150 hours, and Binzel's (1987) data - 1296 hours, but still shorter than the upper limit derived by Ostro et al. (2001) - 2100 hours. However, the two previous values for the period were derived with the assumption of a typical, two maxima and two minima per period lightcurve whereas our composite lightcurve shows a much more complicated picture.
The slow rotation and small diameter of Glauke makes it a primary candidate for tumbling motion, as shown by Harris (1994). Fourier analysis of the lightcurve of Glauke reveals several frequencies, the most significant of which is connected with a period of 26 days. This is roughly half of the value obtained by Harris (46 to 50 or 54 days) and one third of the period derived in this paper ( 77 days). Because of small number of points in our lightcurve it is difficult to tell if other Fourier frequencies of smaller amplitudes are real or are due to noise.

According to Kryszczyńska et al. (1999), Fourier components present in the lightcurve of a freely precessing asteroid may not reflect directly periods of rotation and precession. This fact is seen in case of 4179 Toutatis (Spencer et al. 1995) whose lightcurve does not show any frequency resulting from the period of rotation of 5.4 days. What it shows, however, are the frequencies of precession and the combination of precession and rotation. Radar is much better in such cases - if a sufficiently strong echo is received, it allows one to determine unambigiously both periods.

In the spring of 2000, Glauke was observed by radar (Ostro et al. 2001). As those observations were made in two runs of three consecutive days each, we can assume radar measurements were obtained on Mar. 10 and 20 (these dates are marked in Fig. 3 by vertical lines). Even though our composite lightcurve is not well sampled on these two days we can notice that Glauke appears brighter on Mar. 20 then on Mar. 10. In the absence of larger changes of the geometry of viewing and illumination, this in turn means the projected cross-section area of this asteroid should be bigger on Mar. 20.

According to Ostro et al. (2001), the instantaneous bandwidth of echo from the rotating object, measured for the Arecibo $2380 \mathrm{MHz}$ transmitter, is:

$B=27.7 D \cos (\delta) / P$,

where $P$ is the apparent rotation period (in hours), $\delta$ - the angle between the line of sight and the asteroid's apparent equatorial plane, $D$ - the apparent breadth (in $\mathrm{km}$ ) of the asteroid's poleon silhouette. In most cases, the bandwidth $B$ increases when the cross-section area becomes bigger. If so, then from our previous analysis the observed radar bandwidth $B$ on Mar. 20 should be bigger than that on Mar. 10. This effect is indeed visible in the radar echo spectra (see Fig. 1 in Ostro et al. 2001) but due to noise it lacks statistical significance.

On the other hand, if during the 10 day period the asteroid's instaneneous axis of rotation did not change its position in space significantly, the observed bandwidth could be related to the period of rotation about this axis. If this is the case, then the basic frequency (connected with the period of 26 days) resulting from Fourier analysis of our lightcurve can be interpreted as half of the rotation period of Glauke. The changes of the lightcurve amplitude from cycle to cycle would then be caused by precession of the asteroid axis.

\section{Future observations}

Photometric observations of Glauke are difficult because of very slow changes of its brightness. However, by careful planning, one can obtain useful data to definitely check whether this 


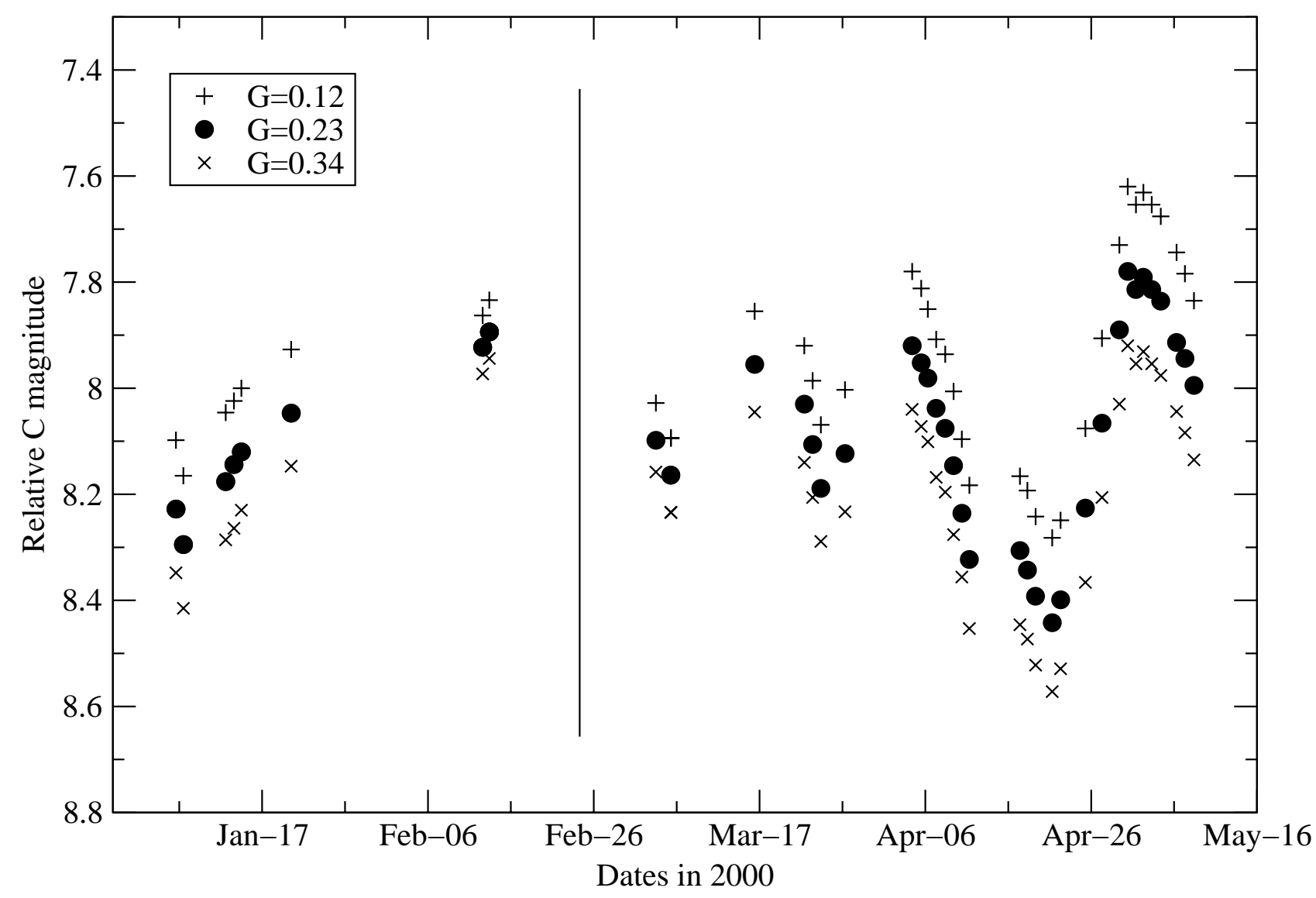

Fig. 2. Lightcurve of 288 Glauke in 2000 apparition, corrected to zero phase angle with $G=0.23$. The vertical line separates two parts not connected in magnitude. To show the influence of $G$ on the brightness of asteroid, additional lightcurves are presented for $G=0.12$ and $G=0.34$.

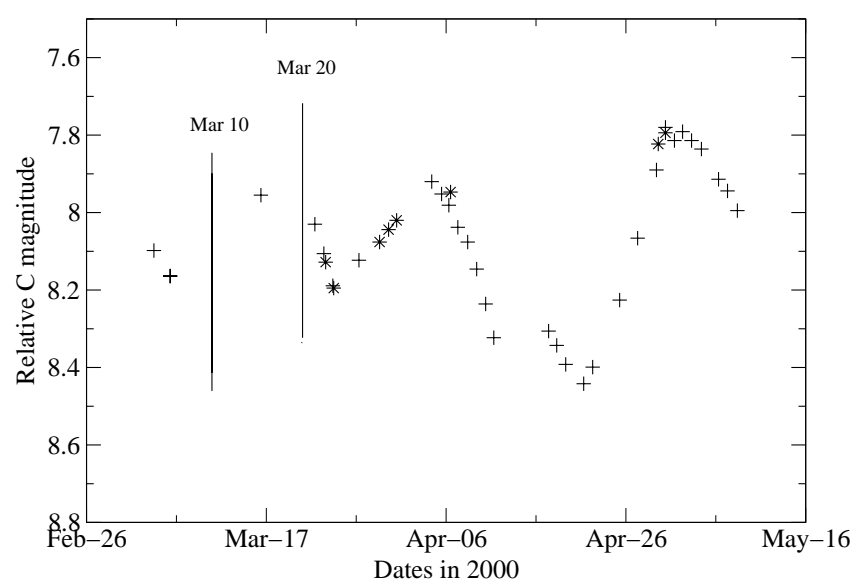

Fig. 3. Composite lightcurve of 288 Glauke, not corrected for lighttime. This lightcurve was done by a simple shift by 77 days of the first part (stars) in time. Vertical lines presents moments of time of radar observations.

object is indeed a tumbling asteroid. This applies particularly to the selected stationary points, where Glauke would be in the same position as during previous observations. In such cases its brightness variations would be observed at similar viewing and illumination geometries, which - in the case of principal axis rotation - should allow one to make one composite lightcurve
Table 2. Geometries of the previous and future observations of 288 Glauke.

\begin{tabular}{cccc}
\hline \hline Date & $\begin{array}{c}\text { Phase } \\
\text { angle }\end{array}$ & $\lambda$ & $\beta$ \\
& $(\mathrm{J} 2000)$ \\
& $\left({ }^{\circ}\right)$ & $\left({ }^{\circ}\right)$ & $\left({ }^{\circ}\right)$
\end{tabular}

\begin{tabular}{cccc}
\hline \multicolumn{4}{c}{ Previous observations } \\
\hline 1982 Apr. 20 & 4 & 207 & 8 \\
1982 May 24 & 17 & 202 & 7 \\
\hline 1984 Sep. 24 & 12 & 45 & -6 \\
1984 Nov. 01 & 2 & 39 & -6 \\
1984 Nov. 27 & 9 & 34 & -6 \\
\hline 2000 Jan. 06 & 19 & 158 & 2 \\
2000 Feb. 12 & 5 & 154 & 4 \\
2000 Apr. 13 & 19 & 146 & 5 \\
2000 May 07 & 26 & 149 & 4 \\
\hline \multicolumn{5}{c}{ Future stationary points } \\
\hline 2005 Jun. 18 & 18 & 223 & 6 \\
2008 Jan. 02 & 16 & 38 & -5 \\
2008 Dec. 18 & 17 & 138 & 0 \\
\hline
\end{tabular}

from both old and new observations. This of course would not be possible in the case of a tumbling body. 
Studies of future oppositions of Glauke in this decade show that during three stationary points the asteroid will be seen in the sky close to the positions it occupied during observations in 1982, 1984 and 2000 (see Table 2). By repeating observations in 2005 and/or 2008 it should be possible to provide a final check of the hypothesis of tumbling rotation of this asteroid.

Acknowledgements. AK was supported by the Polish KBN Grant 2 P03D 007 22, TM by the Polish KBN Grant 2 P03D 007 18, and TK by the Polish KBN Grant 5 P03D 002 20. We are grateful to R. Hirsch and P. Bartczak for their help in the observing campaign. The reduction of all CCD data was performed with the CCLR STARLINK package. We would like to thank A. Schwarzenberg-Czerny for allowing us to use his software for Fourier analysis of unequaly spaced data.

\section{References}

Binzel, R. P. 1987, Icarus, 72, 135

Harris, A. W. 1994, Icarus, 107, 209
Harris, A. W., Young, J. W., Bowell, E., \& Tholen, D. J. 1999, Icarus, 142,173

Henden, A. A. 2000, J. Am. Assoc. Var. Star Obs., 29, 35

Hudson, S., \& Ostro, S. J. 1995, Science, 270, 84

Kryszczyńska, A., Kwiatkowski, T., Breiter, S., \& Michałowski, T. 1999, A\&A, 345, 643

Mottola, S., Sears, W. D., Erikson, A., et al. 1995, Planet. Space. Sci., 43, 1609

Nakamura, T., \& Fuse, T. 1998, Publ. Astron. Soc. Japan, 50, 531

Ostro, S. J., Hudson, R. S., Rosema, K. D., et al. 1999, Icarus, 137, 122

Ostro, S. J., Nolan, M. C., Margot, J. L., et al. 2001, Icarus, 152, 201

Pravec, P., Wolf, M., \& Sarounova, L. 1998, Icarus, 136, 124

Pravec, P., Sarounova, L., Benner, L. A. M., et al. 2000, Icarus, 148, 589

Spencer, J. R., Akimov, L. A., Angeli, C., et al. 1995, Icarus, 117, 71

Veverka, J. P., Thomas, J. P., Harch, A., et al. 1997, Science, 278, 2109

Weidenschilling, S. J., Chapman, C. R., Davis, D. R., et al. 1990, Icarus, 86, 402 